University of Wollongong

Research Online

Faculty of Engineering and Information

Faculty of Engineering and Information

Sciences - Papers: Part B

Sciences

2016

An analytical approximation formula for European option pricing under a new stochastic volatility model with regime-switching

Xinjiang He

University of Wollongong, xinjiang@uow.edu.au

Song-Ping Zhu

University of Wollongong, spz@uow.edu.au

Follow this and additional works at: https://ro.uow.edu.au/eispapers1

Part of the Engineering Commons, and the Science and Technology Studies Commons

Research Online is the open access institutional repository for the University of Wollongong. For further information contact the UOW Library: research-pubs@uow.edu.au 


\title{
An analytical approximation formula for European option pricing under a new stochastic volatility model with regime-switching
}

\author{
Abstract \\ In this paper, an analytical approximation formula for pricing European options is obtained under a newly \\ proposed hybrid model with the volatility of volatility in the Heston model following a Markov chain, the \\ adoption of which is motivated by the empirical evidence of the existence of regime-switching in real \\ markets. We first derive the coupled PDE (partial differential equation) system that governs the European \\ option price, which is solved with the perturbation method. It should be noted that the newly derived \\ formula is fast and easy to implement with only normal distribution function involved, and numerical \\ experiments confirm that our formula could provide quite accurate option prices, especially for relatively \\ short-tenor ones. Finally, empirical studies are carried out to show the superiority of our model based on \\ S\&P 500 returns and options with the time to expiry less than one month.

\section{Disciplines} \\ Engineering | Science and Technology Studies

\section{Publication Details} \\ He, X. \& Zhu , S. (2016). An analytical approximation formula for European option pricing under a new \\ stochastic volatility model with regime-switching. Journal of Economic Dynamics and Control, 71 77-85.
}

This journal article is available at Research Online: https://ro.uow.edu.au/eispapers $1 / 282$ 


\title{
An analytical approximation formula for European option pricing under a new stochastic volatility model with regime-switching
}

\author{
Xin-Jiang HE * $\quad$ Song-Ping ZHU ${ }^{\dagger}$
}

\begin{abstract}
In this paper, an analytical approximation formula for pricing European options is obtained under a newly proposed hybrid model with the volatility of volatility in the Heston model following a Markov chain, the adoption of which is motivated by the empirical evidence of the existence of regime-switching in real markets. We first derive the coupled PDE (partial differential equation) system that governs the European option price, which is solved with the perturbation method. It should be noted that the newly derived formula is fast and easy to implement with only normal distribution function involved, and numerical experiments confirm that our formula could provide quite accurate option prices, especially for relatively short-tenor ones. Finally, empirical studies are carried out to show the superiority of our model based on S\&P 500 returns and options with the time to expiry less than one month.
\end{abstract}

\section{AMS(MOS) subject classification.}

Keywords. European option, Regime-switching Heston model, Perturbation method, Empirical studies.

\footnotetext{
*School of Mathematics and Applied Statistics, University of Wollongong NSW 2522, Australia

†Corresponding author. School of Mathematics and Applied Statistics, University of Wollongong NSW 2522, Australia.
} 


\section{Introduction}

Although the well-known Black-Scholes (B-S) model enjoys great popularity in the area of option pricing due to its simplicity and tractability, a lot of evidence has shown that it is inadequate to precisely model the dynamics of the underlying price. In real markets, the log-returns of the underlying price tend to be skew and fat-tailed [27], and the implied volatility extracted from market data often forms a "smile" curve [10], which is at odds with the constant volatility assumption. Hence, research interest has been led into finding more appropriate models for the underlying price.

In particular, one common modification to the B-S model is to relax the constant volatility assumption. Local volatility and stochastic volatility are two main approaches in the literature. Local volatility was proposed by Dupire [11], who assumed the volatility be a deterministic function of the underlying price and time. However, stochastic volatility models are much more favored since the "smile dynamics" are poorly captured by local volatility models [16].

A number of authors has worked on stochastic volatility models. The addition of another stochastic source has made the option pricing problem much more complicated, and in most cases only numerical methods could be used to find the European option prices (e.g., [28],[34]). Moreover, although Hull \& White [21] were able to derive a semi-closed form pricing formula under their model, their assumption that the volatility be independent of the underlying price is inappropriate since many empirical studies have shown that the volatility and the underlying price are negatively related [2]. A similar case is the SteinStein model [31], where a closed-form formula for European options exists. However, their volatility dynamic is again inconsistent with the fact that the volatility should always be positive. Fortunately, the famous Heston model [19] was proposed in 1993, the volatility dynamic of which satisfies a wide range of properties, such as the non-negative property and mean-reverting property, and most importantly, a closed-form pricing formula could be 
worked out for European options. However, it should also be pointed out that the Heston model is not perfect either since there already exists empirical evidence showing that the mean-reverting property for a volatility process is actually not linear [3].

In this paper, we propose a new stochastic volatility model, where the volatility of volatility in the Heston model follows a Markov chain so that it could jump between two states. Our motivation stems from the fact that the Heston model with constant parameters may not provide good fit to market data, and the time-dependent Heston model has already been studied by Buhler [5], Mikhailov \& Nogel [26] and so on. Moreover, the stochastic volatility of volatility has been considered in modeling the VIX dynamics [23], while variance and volatility swaps are priced under the regime-switching Heston model with the long-term mean following a Markov chain in [13]. In fact, quite a lot of empirical evidence already suggests that there are certain advantages to incorporate regimeswitching into the volatility process. For example, Kalimipalli \& Susmel [24] adopted a regime-switching stochastic volatility model to describe the short-term interest rate and they found that it can lead to a better in-sample performance than the classical stochastic volatility model, while results in [22] show that squared returns are better specified by regime-switching stochastic volatility models. Also, it has already been shown by So et al. [30] that persistence in volatility can be explained by a regime-switching stochastic volatility model. In addition, Vo [32] found strong evidence of regime-switching in real markets, and he even pointed out that introducing regime-switching into the framework of stochastic volatility can bring two main advantages; one is that it can significantly enhance the forecasting power of the stochastic volatility model, and another is that the regimeswitching stochastic volatility model does a better job in capturing major events affecting the market. Therefore, considering the fact that the Heston stochastic volatility model is a widely adopted model in real markets, it is natural for us to consider an introduction of regime-switching into this particular model, with the volatility of volatility in the Heston model being made regime-switching. Of course, since a great feature of the Heston model is 
the existence of a simple and analytical pricing formula for European options, the challenge we face is how to keep the tractability of the new model, which is more complicated than the Heston model. In this paper, we present one way to preserve the tractability by deriving an approximation formula for European option prices at the expense of imposing a restriction that the formula is only suitable for short-tenor European options. In the following, this analytical approximation formula will be derived based on the perturbation method, which has been widely adopted in solving option pricing problems [20, 33, 35]. It should be noticed that our pricing formula is rather simple with only normal distribution function involved and is suitable for options with short tenors, which are dominated in real markets anyway. Finally, to show the performance of our model in real markets, empirical studies are carried out to compare our model and the Heston model with S\&P 500 returns and short-tenor options. Results confirm that our model generally outperforms the Heston model, and thus it could be used as an alternative to the Heston model for some markets.

The rest of the paper is organized as follows. In Section 2, we will first briefly introduce our new stochastic volatility model and present the coupled PDE (partial differential equation) system for European options, after which the perturbation method is applied to find pricing formula for options with short tenors. In Section 3, numerical experiments are carried out to show the accuracy of the approximation involved in our newly derived formula. In Section 4, empirical studies are carried out to show the performance of our newly proposed model, followed by some concluding remarks given in the last section.

\section{European options with short tenors}

In this section, the dynamics of the regime-switching Heston model are presented and the coupled PDE system governing the European put option prices is given, followed by the approximation formula obtained through the perturbation method.

Let $S$ and $v$ be the underlying price and the volatility respectively, the regime-switching 
Heston model under the risk-neutral measure is specified as

$$
\begin{aligned}
\frac{d S}{S} & =r d t+\sqrt{v_{t}} d W_{t}^{1}, \\
d v_{t} & =k\left(\theta-v_{t}\right) d t+\sigma_{X_{t}} \sqrt{v_{t}} d W_{t}^{2}
\end{aligned}
$$

where $W_{t}^{1}$ and $W_{t}^{2}$ are two standard Brownian motions with correlation $\rho . r$ is the riskneutral interest rate. $k$ and $\theta$ are the mean-reverting speed and level respectively. $\sigma_{X_{t}}$ is the volatility of volatility controlled by the Markov chain $X_{t}$, which is independent of $W_{t}^{1}$ and $W_{t}^{2}$. In particular, $X_{t}$ is defined as

$$
X_{t}= \begin{cases}1, & \text { when the economy is believed to be in State 1, } \\ 2, & \text { when the economy is believed to be in State 2 }\end{cases}
$$

with the transition between the two states following a Poisson process as

$$
P\left(t_{i j}>t\right)=e^{-\lambda_{i j} t}, i, j=1,2, i \neq j .
$$

Here $\lambda_{i j}$ is the transition rate from State $i$ to $j$, and $t_{i j}$ is the time spent in State $i$ before transferring to State $j$. Apparently, when the two transition rates $\lambda_{12}$ and $\lambda_{21}$ take the value of zero, our model would surely degenerate to the Heston model.

Following [12], the state space of $X_{t}$ could be taken to be the set of unit vectors $\left\{e_{1}, e_{2}\right\}$, with $e_{1}=(1,0)^{\prime}$ and $e_{2}=(0,1)^{\prime}$. Here, $v^{\prime}$ denotes the transpose of the vector $v$. Then, if we let the European put option price vector be denoted by

$$
\widetilde{U}(S, v, t)=\left(U\left(S, v, t, e_{1}\right), U\left(S, v, t, e_{2}\right)\right)^{\prime},
$$

it is not difficult to find that $U\left(S, v, t, X_{t}\right)=<\widetilde{U}(S, v, t), X_{t}>$, where $<\cdot, \cdot>$ denotes the 
inner product of two vectors, which could certainly yield

$$
d U=<d \widetilde{U}, X_{t}>+<\widetilde{U}, d X_{t}>
$$

By applying the Itô lemma to $U$, the following equation can be obtained

$$
\begin{aligned}
d U & =\frac{\partial U}{\partial t} d t+\frac{\partial U}{\partial v} d v+\frac{\partial U}{\partial S} d S+\frac{1}{2} \frac{\partial^{2} U}{\partial S^{2}}(d S)^{2}+\frac{1}{2} \frac{\partial^{2} U}{\partial v^{2}}(d v)^{2} \\
& +\frac{\partial^{2} U}{\partial S \partial v} d S d v+<\widetilde{U}, d X_{t}>
\end{aligned}
$$

Considering the fact that the discounted option price $e^{-r t} U$ should be a martingale, the coupled PDE system can be derived as

$$
\begin{aligned}
\frac{\partial U_{1}}{\partial t} & +\frac{1}{2} v S^{2} \frac{\partial^{2} U_{1}}{\partial S^{2}}+r S \frac{\partial U_{1}}{\partial S}-r U_{1} \\
& +\frac{1}{2} \sigma_{1}^{2} v \frac{\partial^{2} U_{1}}{\partial v^{2}}+\sigma_{1} v \rho S \frac{\partial^{2} U_{1}}{\partial S \partial v}+k(\theta-v) \frac{\partial U_{1}}{\partial v}-\lambda_{12}\left(U_{1}-U_{2}\right)=0
\end{aligned}
$$

and

$$
\begin{aligned}
\frac{\partial U_{2}}{\partial t} & +\frac{1}{2} v S^{2} \frac{\partial^{2} U_{2}}{\partial S^{2}}+r S \frac{\partial U_{2}}{\partial S}-r U_{2} \\
& +\frac{1}{2} \sigma_{2}^{2} v \frac{\partial^{2} U_{2}}{\partial v^{2}}+\sigma_{2} v \rho S \frac{\partial^{2} U_{2}}{\partial S \partial v}+k(\theta-v) \frac{\partial U_{2}}{\partial v}-\lambda_{21}\left(U_{2}-U_{1}\right)=0
\end{aligned}
$$

where $U_{1}=U\left(S, v, t, e_{1}\right), U_{2}=U\left(S, v, t, e_{2}\right)$. The terminal condition for a put option ${ }^{1}$ is

$$
U_{i}(S, v, T)=\max (K-S, 0),
$$

\footnotetext{
${ }^{1}$ It suffices to derive a pricing formula for European put options. As for the call, we can just use the put-call parity to derive the formula.
} 
and the boundary conditions are

$$
\begin{aligned}
& \lim _{S \rightarrow 0} U_{i}(S, v, T)=K e^{-r(T-t)}, \\
& \lim _{S \rightarrow+\infty} U_{i}(S, v, T)=0, \\
& \lim _{v \rightarrow 0} U_{i}(S, v, T)=\max \left(K e^{-r(T-t)}-S, 0\right), \\
& \lim _{v \rightarrow+\infty} \frac{\partial U_{i}(S, v, T)}{\partial v}=0,
\end{aligned}
$$

for $i=1,2$. The boundary conditions along the $v$ direction are discussed in [36].

To solve the above PDE system with the perturbation method, a small parameter should be introduced. As most of the options traded in real markets are short-tenor, it is reasonable to assume that the time to maturity is small. As a result, we let $\tau=T-t$, and assume $\tau=\epsilon T$, where $\epsilon$ is a small parameter. Moreover, following Zhu \& Chen [35], we also introduce a scaled parameter $x=\frac{S-K}{\sqrt{\epsilon} K}$, so that the PDE system (2.3)-(2.4) could be converted into

$$
\begin{aligned}
\frac{\partial U_{1}}{\partial T} & =\frac{1}{2} v(\sqrt{\epsilon} x+1)^{2} \frac{\partial^{2} U_{1}}{\partial x^{2}}+r(\sqrt{\epsilon} x+1) \sqrt{\epsilon} \frac{\partial U_{1}}{\partial x}+\sigma_{1} \rho v(\sqrt{\epsilon} x+1) \sqrt{\epsilon} \frac{\partial^{2} U_{1}}{\partial x \partial v} \\
& +\frac{1}{2} \sigma_{1}^{2} v \epsilon \frac{\partial^{2} U_{1}}{\partial v^{2}}+k(\theta-v) \epsilon \frac{\partial U_{1}}{\partial v}-r \epsilon U_{1}-\lambda_{12} \epsilon\left(U_{1}-U_{2}\right),
\end{aligned}
$$

and

$$
\begin{aligned}
\frac{\partial U_{2}}{\partial T} & =\frac{1}{2} v(\sqrt{\epsilon} x+1)^{2} \frac{\partial^{2} U_{2}}{\partial x^{2}}+r(\sqrt{\epsilon} x+1) \sqrt{\epsilon} \frac{\partial U_{2}}{\partial x}+\sigma_{2} \rho v(\sqrt{\epsilon} x+1) \sqrt{\epsilon} \frac{\partial^{2} U_{2}}{\partial x \partial v} \\
& +\frac{1}{2} \sigma_{2}^{2} v \epsilon \frac{\partial^{2} U_{2}}{\partial v^{2}}+k(\theta-v) \epsilon \frac{\partial U_{2}}{\partial v}-r \epsilon U_{2}-\lambda_{21} \epsilon\left(U_{2}-U_{1}\right) .
\end{aligned}
$$

In order to eliminate the factor $\sqrt{\epsilon} K$ in the initial condition, we set

$$
P_{i}(x, v, T)=U_{i}(x, v, T) /(\sqrt{\epsilon} K), i=1,2,
$$


and expand $P_{i}$ in terms of $\sqrt{\epsilon}$ as

$$
P_{i}=P_{i}^{0}+\sqrt{\epsilon} P_{i}^{1}+O(\epsilon), i=1,2 .
$$

By substituting Equation (2.7) into the system (2.5)-(2.6) and eliminate the $O(1)$ term, we can obtain

$$
\begin{aligned}
& \frac{\partial P_{i}^{0}}{\partial T}=\frac{1}{2} v \frac{\partial^{2} P_{i}^{0}}{\partial x^{2}}, \\
& P_{i}^{0}(x, v, 0)=\max (-x, 0),
\end{aligned}
$$

for $i=1,2$. It should be noted that $v$ can be regarded as a fixed constant when solving the newly obtained PDEs (2.8) since it does not involve the partial differential derivative with respect to $v$ is not involved in the differential operator. As a result, it is clear that both of the newly obtained PDEs are homogeneous heat equation defined along the real axes, which can be solved with the fundamental solution as

$$
\begin{aligned}
P_{i}^{0} & =\frac{1}{\sqrt{2 v \pi T}} \int_{-\infty}^{+\infty} e^{-\frac{(x-\xi)^{2}}{2 v T}} d \xi \\
& =\sqrt{\frac{v T}{2 \pi}} e^{-\frac{x^{2}}{2 v T}}-x\left[1-N\left(\frac{x}{\sqrt{v T}}\right)\right] .
\end{aligned}
$$

In a similar fashion, we could obtain the PDE for $P_{i}^{1}, i=1,2$ by collecting the coefficients of $\sqrt{\epsilon}$ together, which is

$$
\begin{aligned}
& \frac{\partial P_{i}^{1}}{\partial T}=\frac{1}{2} v \frac{\partial^{2} P_{i}^{1}}{\partial x^{2}}+x v \frac{\partial^{2} P_{i}^{0}}{\partial x^{2}}+r \frac{\partial P_{i}^{0}}{\partial x}+\rho \sigma_{i} v \frac{\partial^{2} P_{i}^{0}}{\partial x \partial v}, \\
& P_{i}^{1}(x, v, 0)=0
\end{aligned}
$$

for $i=1,2$. It should be noticed that PDE (2.9) is an inhomogeneous heat equation with homogeneous initial condition, which can be solved with green function. However, the calculation is rather complicated and thus alternative ways attempted. Luckily, one way 
would lead to much simpler formula, which is illustrated in the following. According to the results in [20], if $C_{t}-\frac{1}{2} C_{x x}=0$, and $D_{t}-\frac{1}{2} D_{x x}=v C$, then a particular solution is $D=C v t$. In addition, if $C_{t}-\frac{1}{2} C_{x x}=0$ and $D_{t}-\frac{1}{2} D_{x x}=x v C$, then a particular solution is $D=x v t C+\frac{1}{2}(v t)^{2} C_{x}$. As a result, considering the situation that $\frac{\partial P_{i}^{0}}{\partial x}, \frac{\partial^{2} P_{i}^{0}}{\partial x^{2}}$ and $\frac{\partial^{2} P_{i}^{0}}{\partial x \partial v}$ also satisfy the equation $C_{t}-\frac{1}{2} C_{x x}=0$, a particular solution for $P_{i}^{1}$ could be obtained as

$$
\begin{aligned}
P_{i}^{1} & =\left[\frac{r}{v} \frac{\partial P_{i}^{0}}{\partial x}+\rho \sigma_{i} \frac{\partial^{2} P_{i}^{0}}{\partial x \partial v}\right] v T+\frac{\partial^{2} P_{i}^{0}}{\partial x^{2}} x v T+\frac{1}{2}(v T)^{2} \frac{\partial^{3} P_{i}^{0}}{\partial x^{3}} \\
& =-r T\left[1-N\left(\frac{x}{\sqrt{v T}}\right)\right]-\frac{\rho \sigma_{1} v T x}{2 \sqrt{2 \pi v T}} e^{-\frac{x^{2}}{2 v T}}+\frac{x v T}{2 \sqrt{2 \pi v T}} e^{-\frac{x^{2}}{2 v T}}
\end{aligned}
$$

for $i=1,2$. Therefore, after some algebraic manipulation, we can arrive at the final result written in the original parameters

$$
\begin{aligned}
U_{i}(S, v, \tau) & =\sqrt{\epsilon} K P_{i}=\sqrt{\epsilon} K\left[P_{i}^{0}+\sqrt{\epsilon} P_{i}^{1}+O(\epsilon)\right] \\
& =\left[\frac{\sqrt{v \tau}}{2 \sqrt{2 \pi}}(S+K)-\frac{\rho \sigma_{i} \sqrt{v \tau}}{2 \sqrt{2 \pi}}(S-K)\right] e^{-\frac{(S-K)^{2}}{2 v \tau K^{2}}} \\
& -[S+(r \tau-1) K]\left[1-N\left(\frac{S-K}{\sqrt{v \tau} K}\right)\right]+O(\tau), \quad i=1,2 .
\end{aligned}
$$

Obviously, the newly obtained formula is rather simple, which only involves the calculation of the normal distribution function, and thus the implementation of this formula can be as easy as the B-S formula. This is a great advantage as the simplicity of a pricing formula presents enormous benefits in terms of model calibration as well as in line with the increasing demand of super fast computation of option price as a result of recent trend of algorithm trading. It should be pointed out that the mean-reversion speed $k$ and the long-term mean $\theta$ of the volatility do not explicitly appear in the final approximation formula for short-tenor options, which implies that the two parameters have little influence on option prices with short time to expiry. This is also the main reason why we make $k$ and $\theta$ the same for both regimes. It should also be noted that the small parameter $\epsilon$ does not explicitly appear in the final approximation formula, as it is a parameter determined 
by the tenor of a contract and used to judge the validity of the approximation. Therefore, all we need is to ensure that the time to expiry of a contact to be priced is small enough so that $\epsilon \ll 1$. Another point that should be emphasized is that although the techniques we employ in the regular perturbation are straightforward, proposing a new solution technique is not what we were trying to achieve in this paper; proposing a new model by introducing regime-switching into the Heston model that can provide a better empirical performance than the classical Heston model is.

Once an approximation formula for short-tenor options is obtained, it is natural for us to numerically check its accuracy, which would be presented in the next section.

\section{Numerical verification}

In this section, numerical experiments are conducted to show the performance of Formula (2.11) when the time to expiry varies. The accuracy is demonstrated through a comparison of the option prices calculated with the newly derived formula and those obtained by Monte Carlo simulation of SDE (2.1). In the following, the current state is assumed to be 1, and the strike price of the option $K$ is 10 . The volatility of volatility for state 1 and $2, \sigma_{1}$ and $\sigma_{2}$, takes the value of 0.3 and 0.6 respectively. The two transition rates $\lambda_{12}$ and $\lambda_{21}$ are 40 and 60 , respectively. The mean reverting speed $k$ is 2.5 , the long-term mean $\theta$ is 0.16 , and the initial value of the volatility $v_{0}$ is 0.2 . The correlation between the underlying price and the volatility $\rho$ is -0.5 .

Depicted in Figure 1 are the option prices for different underlying prices when time to expiry is one month, and it is clear that the results obtained from our approximation agrees very well with the Monte Carlo prices. Specifically, the point-wise absolute error in Figure 1.1 less than 0.01, which demonstrates that our approximation is very accurate when the time to expiry is relatively short. On the other hand, When the time to expiry increases to six months, our approximation is still quite close to the Monte Carlo price as shown in 


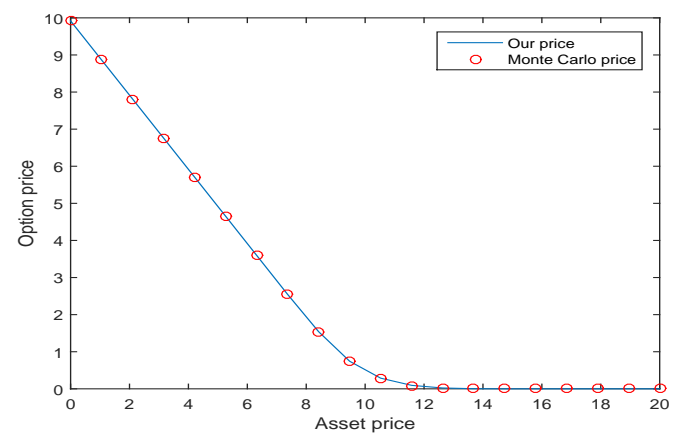

(a) $\tau=1 / 12, r=0.1$.

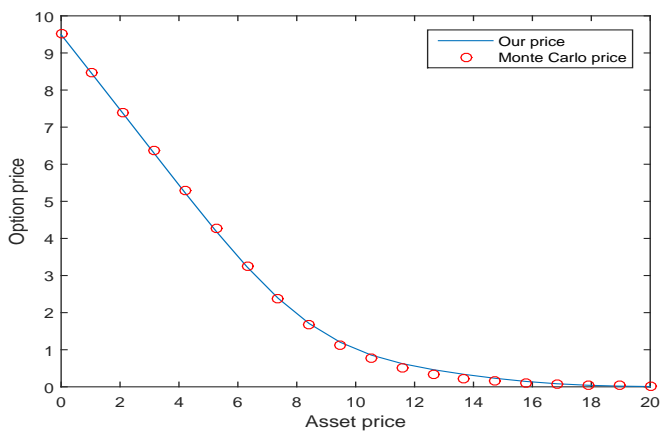

(b) $\tau=1 / 4, r=0.1$.

Figure 1: Our price vs Monte Carlo price with different underlying prices.

Figure 1.2, though the performance of our approximation this time is clearly worse than the case when the time to expiry is short. This is expected since the approximation is made based on the assumption of small time to maturity. It is also interesting to notice that our approximation performs slightly better for in the money option than the out of money option.

With the confidence of our pricing formula for short-tenor options, empirical studies are carried out with the time to expiry of the chosen options being less than one month to make the comparison of our model and the well-known Heston model, which is a widely adopted stochastic volatility model in real markets, with the results presented in the next section.

\section{Empirical studies}

In this section, empirical results are presented and discussed with the Heston model being taken as a benchmark to assess the performance of our model in real markets. In the following, we will firstly describe the data we use and introduce the method adopted for parameter estimation. Then, the performance of these two models is quantitatively compared by the pricing errors measured with the "distance" between model-produced and market prices, and it is widely accepted that a model is regarded as the better one 
if it exhibits less pricing errors. In fact, pricing errors usually consist of two parts: the so-called in-sample errors and out-of-sample errors. Conventionally, the period in which market data is available is divided into two; the first one refers to a period in which data is used for parameter determination (referred to as the "in-sample observations") and the second one then refers to the period in which data is used to verify the performance of a model through a comparison between the observed option prices and the calculated option prices based on the parameters determined from the "in-sample" period (in contrast to the first period, data in the second period is usually referred to as the "out-of-sample observations"). It should be remarked that it is usually difficult to achieve lower in- and out-of-sample errors simultaneously. When comparing two models through a comparison of the in- and out-of-sample errors, it is hard to draw a conclusion if only one part of errors is less than the other. However, there should be no doubt in one's mind which model is superior if both of its in- and out-of-sample fitness are better. This is the principle based on which we draw the conclusions of our empirical studies in the subsection 4.3.

\subsection{Data description}

Our empirical study is conducted on a data set of the S\&P 500 Index and European call options written on the S\&P 500 Index from Jan 2011 to Jun $2011^{2}$. However, raw data should not be adopted directly in the estimation since sample noise needs to be eliminated. Hence, two appropriate filters presented below were applied to the raw data, before they were used to estimate model parameters.

First of all, following a number of authors, such as Bakshi et. al [2] and Christoffersen et. al [7], only Wednesday and Thursday options data is adopted. In particular, Wednesday options data is used in estimation since Wednesday is least likely to be a holiday in a week and also less likely to be affected by the "day-of-the-week" effect than other days such

\footnotetext{
${ }^{2}$ Since our formula could provide satisfactory accuracy for short-tenor options, all options used are to expire within one month.
} 
as Monday and Friday, whereas the corresponding Thursday data serves as the market price to be compared with the predicted price calculated by the estimated parameters. Another motivation for only using Wednesday data in parameter estimation is that global optimization problems are usually quite time-consuming and choosing one day a week allows us to study a relatively longer time series yielding more reliable results. Secondly, very deep in-the-money and very deep out-of-money options are discarded due to their inactivity in the market and may also have liquidity-related biases [29]. Specifically, options with the absolute moneyness, defined as the relative difference between the S\&P 500 Index

value and the corresponding strike price (Moneyness $=\frac{S-K}{K}$ ) over $10 \%$ are excluded.

It should be noted that after these filters are applied, more efficiency can be achieved in the process of parameter estimation with all the important information still preserved according to various previously conducted empirical studies in the literature $[2,7]$.

As for the risk-free interest rate, we choose the one-month U.S. Treasury Bill Rate, which is released daily, as a proxy of the risk-free rate $[4,29]$ since the time to expiry of the selected options is less than 30 days. Upon the preparation of all the data described above, parameter estimation was conducted, following a simple flow chart (cf. Figure 2) exhibiting the specific steps in our empirical studies. Clearly, adopting a genetic algorithm as the main tool of optimization forms the core of the determination of model parameters, the details of which are described in the next subsection.

\subsection{Parameter estimation}

In this section, we first provide a brief summary of the model parameters that need to be determined in both models and then introduce a genetic algorithm used to determine model parameters. 


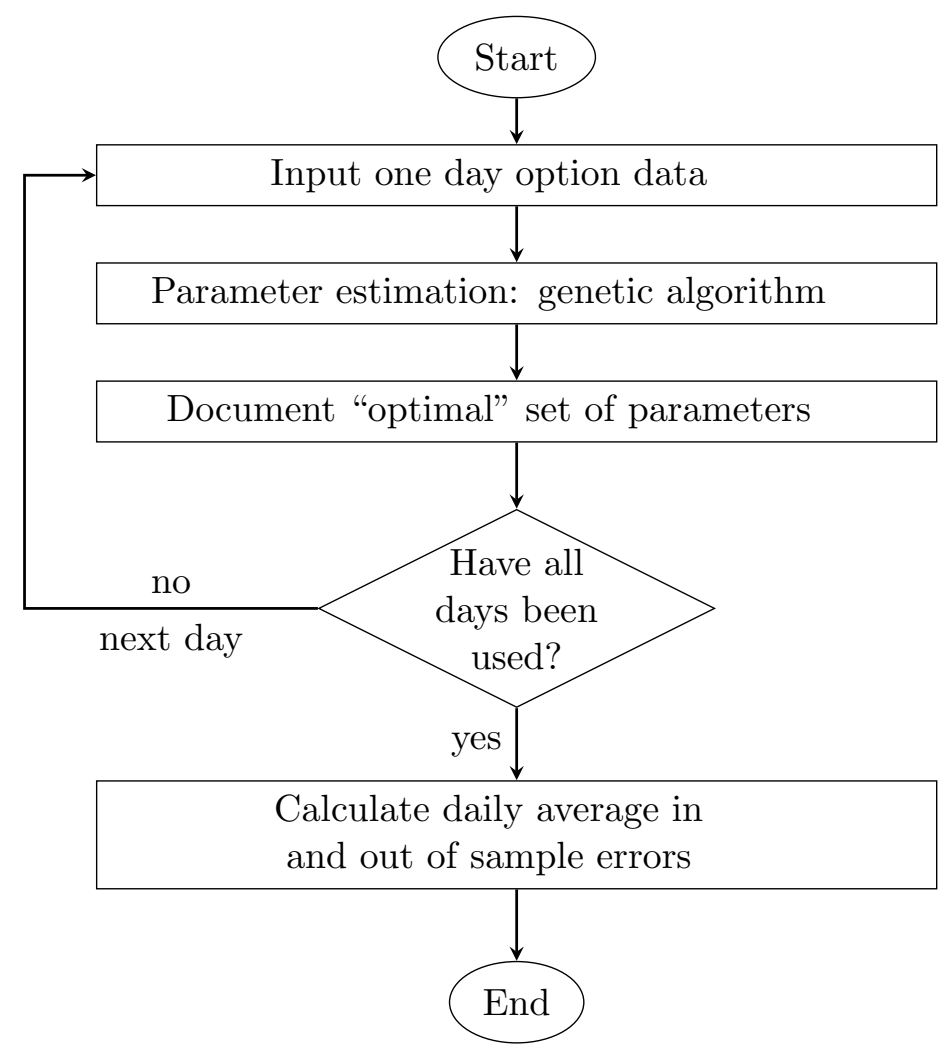

Figure 2: Flow chart on how our empirical studies are conducted.

Recall that the Heston model is specified as

$$
\begin{aligned}
\frac{d S}{S} & =r d t+\sqrt{v} d W_{t} \\
d v & =k(\theta-v) d t+\sigma \sqrt{v} d B_{t}
\end{aligned}
$$

where the following five parameters, i.e. the mean-reversion speed $k$, the long-term mean $\theta$, the volatility of volatility $\sigma$, correlation $\rho$ between $W_{t}$ and $B_{t}$ and the initial value of volatility $v_{0}$, need to be determined from real market data, before the Heston pricing formula could be used to calculate the "fair" price of an option.

On the other hand, it should be pointed out that it is usually very difficult to determine which state the current underlying price belongs to in real markets. Thus, following [17], it is more reasonable to assume that the state is unobservable and regard the probability of each state at the point when the option contact needs to be priced as another parameter 
that needs to be estimated. To be more specific, if we let $\pi$ denote the probability of the underlying price belonging to State 1 , it is not difficult to find that the option price $U^{\text {model }}$ under our newly proposed model should be calculated as

$$
U^{\text {model }}(S, v, \tau)=\pi * U_{1}(S, v, \tau)+(1-\pi) * U_{2}(S, v, \tau)
$$

It should be pointed that there are actually five parameters which need to be estimated, i.e., the probability $\pi$ and the four parameters, $\rho, \sigma_{1}, \sigma_{2}, v_{0}$, in the expression of $U_{1}$ and $U_{2}$. In other words, the size of parameter space remains the same as that of the Heston model. Specifically, the mean-reversion speed $k$ and the long-term mean $\theta$ in the Heston model have disappeared in the new formula as a result of the restriction we imposed for pricing short-tenor contacts only, while the probability $\pi$ and the two parameters representing the volatility of volatility in both regimes, $\sigma_{1}$ and $\sigma_{2}$, now appear in the new formula to capture the regime-switching dynamics. Coincidentally, the fact that the size of the parameter space of the new pricing formula is the same as that of the Heston model gives an additional benefit that there is no extra burden when calibrating our model compared with the classical Heston model, as long as the new formula is only used for short-tenor contracts, which are the most popular cases in financial markets anyway. It should also be stressed that when using the pricing formula (4.2), the parameter estimation process for our model is similar to that for the classical Heston model, and there is no need to conduct separate estimation for the probability $\pi$ and the two values for the volatility of volatility in the two regimes, $\sigma_{1}$ and $\sigma_{2}$. This is because the three parameters can be simultaneously obtained with other model parameters with optimization algorithms, which will be illustrated in the following.

Now we have figured out all the parameters that need to be determined, it is time to find an appropriate approach. A common one is to find the set of "optimal" parameters that minimizes the "distance" between market and model prices. Actually, there are different 
kinds of definition for the distance, a common one is to take the percentage mean squared error (PMSE)

$$
P M S E=\frac{1}{N} \sum_{i=1}^{N}\left[\frac{U^{\text {Market }}-U^{\text {Model }}}{U^{\text {Model }}}\right]^{2},
$$

as the objective distance function to measure the relative difference between market and model prices. Here $U^{\text {Market }}$ denotes the market price of an option contract from one sample, $U^{\text {Model }}$ represents the corresponding calculated price with our pricing formula with a particular set of parameters and $N$ is the total number of observations selected in a single estimation. However, the main disadvantage in choosing such an objective function is that a cheap option (i.e., low $U^{\text {Market }}$ ) could place an abnormally high amount of weight in PMSE. Therefore, following Christoffersen \& Jacobs [6] and Lim \& Zhi [25], we instead chose the dollar mean-squared errors

$$
M S E=\frac{1}{N} \sum_{i=1}^{N}\left[U^{\text {Market }}-U^{\text {Model }}\right]^{2},
$$

as the objective function. In this case, with a given set of data, what we need to do is to use particular techniques to search the parameter space to find a set of parameters that can minimize the MSE between the model price and the market price.

Another issue is how to choose an appropriate optimization method. It should be noticed that although local minimization, which requires an initial guess that is very close to the true optimal solution, can be much less time consuming, its results are usually not reliable since it depends too much on the selected initial guess. In fact, the objective function (4.3) is not necessarily convex and thus there could exist several local minima, which would probably result in a local minimum being taken as the global minimum. In contrast, a properly designed global optimization should able to skip local minima and correctly identify the global minimum in an efficient way.

A genetic algorithm [8] is such a global optimization with some very nice properties. It is based on the idea of natural selection, introducing stochastic factors when searching for a 
satisfactory result in order to skip over local optima. One of the most important reasons for us to adopt this particular algorithm is that it randomly selects a number of different initial guesses to explore the entire solution space. Moreover, a so-called "mutation" step in the optimization process allows the algorithm to avoid local minima by preventing parameters from being restricted to a small region. In the literature, genetic algorithms have been applied in finance by quite a few researchers already. For instance, Gimeno \& Nave [14] conducted estimation of the term structure of interest rates with a genetic algorithm while Grace [15], Cont \& Ben Hamida [9] and He \& Zhu [18] have adopted it in the area of option pricing. It is even pointed out by Bajpai \& Kumar [1] that genetic algorithms are one of the best global optimization methods and can provide high quality solutions since they are intrinsically parallel and can explore the solution space in multiple directions at the same time.

It should be remarked that another advantage of adopting a genetic algorithm is its implementation in the Matlab is straightforward by using a built-in function $g a$. As a result of the numerical implementation, Table 1 exhibits the estimated daily averaged parameters extracted from the selected market data for the two models under consideration in this paper, respectively.

Table 1: Estimated parameters

\begin{tabular}{|c|c|c|c|c|c|c|c|c|}
\hline parameters & $\rho$ & $v_{0}$ & $\sigma_{1}$ & $\sigma_{2}$ & $\pi$ & $\sigma$ & $k$ & $\theta$ \\
\hline Our model & -0.2398 & 0.0218 & 2.0568 & 1.8818 & 0.5049 & & & \\
\hline Heston model & -0.7921 & 0.0666 & & & & 1.7665 & 293.4422 & 0.0542 \\
\hline
\end{tabular}

\subsection{Empirical comparison}

In this subsection, the performance of our model and the Heston model in real markets is compared using the extracted parameters. In terms of model performances, it is widely accepted that a model is regarded better if there exist less accumulated pricing errors calculated by the distance between model prices and market prices. Here, we adopt the 
root mean-squared error (RMSE), which is the square root of the objective function (MSE), as a measure of "goodness of fit". Table 2 exhibits the in- and out-of-sample errors for the two models.

Table 2: In- and out-of-sample errors for the two models

\begin{tabular}{|c|c|c|}
\hline Error & In-sample & out-of-sample \\
\hline Our model & 0.5198 & 0.9221 \\
Heston model & 0.7753 & 1.3911 \\
Relative difference & $67.05 \%$ & $66.29 \%$ \\
\hline
\end{tabular}

It is obvious from Table 2 that our model generally outperforms the Heston model in terms of both in- and out-of-sample errors. To be more specific, from the perspective of in-sample errors, the daily averaged RMSE for our model is only 0.5198, compared with 0.7753 for the Heston model. It is clear that our model is superior to the Heston model in this case since the relative difference ${ }^{3}$ between the two models, as far as the daily averaged RMSE is concerned, is less than $68 \%$, which means that would be over $32 \%$ of improvement if our model is used and is surprisingly quite significant. On the other hand, when out-of-sample errors are taken into consideration, a similar pattern emerges; our model still shows a much better performance than that of the Heston model, and the relative difference between them is even a bit smaller at $66.29 \%$. Therefore, combining both in-sample and out-of-sample observations, we can conclude that our model serves as a better choice than the Heston model for the data set chosen in this comparison. One of the main reasons for such a significant improvement is that the chosen market does possess different regimes. Naturally it is expected that the market dynamics are better captured by a regime-switching stochastic volatility model.

It is also interesting to notice that the RMSE for out-of-sample errors is always much larger than that for in-sample errors. This is not difficult to understand since model

\footnotetext{
${ }^{3}$ The relative difference is defined as

$$
\text { Relative difference }=\frac{\text { Our model error }}{\text { Heston model error }} .
$$
}


prices are calculated with determined parameters. Those obtained with in-sample data are believed to be "closest" to market prices of the corresponding option contracts, which implies that in-sample errors should be relatively low, while out-of-sample data are only used as the verification of option prices and certainly there is no guarantee that out-ofsample errors be low.

On the other hand, options are traded with a wide range of strikes in real markets and thus it is important to check the out-of-sample valuation errors sorted by moneyness, which are shown in Table 3. While the range of moneyness is indicated on the top row of the table, the abbreviation in the parentheses indicate "out of money" "at the money" and "in the money", respectively, from the left to the right columns.

Table 3: Out-of-sample errors according to moneyness

\begin{tabular}{|c|c|c|c|}
\hline Moneyness & $0.90<S / K<0.97(O)$ & $0.97 \leq S / K \leq 1.03(A)$ & $1.03<S / K<1.10(I)$ \\
\hline Our model & 0.4266 & 1.1560 & 0.8171 \\
Heston model & 0.9546 & 1.5983 & 1.3162 \\
Relative difference & $44.69 \%$ & $72.33 \%$ & $62.08 \%$ \\
\hline
\end{tabular}

From this table, it is clear that the performance of our model is better than that of the Heston model in all of the three categories. Although the improvement for at-themoney options is lowest, the daily averaged pricing error for our model are around $72 \%$ of that for the Heston model, which is quite significant. The maximal improvement occurs for the category of out-of-money options, where the relative difference even fall below $50 \%$. Thereby, we can confidently conclude that our model has given an overall better performance than that of the Heston model. Of course, this conclusion is based on the empirical test of one set of data. It is quite possible that the performance of these two models may reverse with some other data sets. However, our empirical study presented here can at least suggest it may offer as a good competitor of the Heston model for short-tenor options in some other markets, such as commodity and futures exchange markets. 


\section{Conclusion}

In this paper, an approximation pricing formula for European options under a newlyproposed stochastic volatility model is presented. The main contributions of our contribution can be summarized into three items: (i) A new stochastic volatility model is proposed with a key feature that the constant volatility of volatility in the original Heston model is now allowed to change randomly following a Markov chain. (ii) An analytical pricing formula for short-tenor European options has been worked out with a key feature that the newly-obtained formula is fast and easy to implement with only normal distribution function involved. (iii) Not only have we tested the reliability and accuracy of the newly-obtained formula, we have also implemented it in an empirical experiment with real market data to demonstrate the superiority of the new model over the classical Heston model; empirical results based on S\&P 500 returns and options demonstrate that our model significantly outperforms the Heston model for the case tested, and our empirical study suggests that the new model has a great potential to be used as an alternative to the Heston model for short-tenor options.

\section{References}

[1] P. Bajpai and M. Kumar. Genetic algorithm-an approach to solve global optimization problems. Indian Journal of computer science and engineering, 1(3):199-206, 2010.

[2] G. Bakshi, C. Cao, and Z. Chen. Empirical performance of alternative option pricing models. The Journal of Finance, 52(5):2003-2049, 1997.

[3] G. Bakshi, N. Ju, and H. Ou-Yang. Estimation of continuous-time models with an application to equity volatility dynamics. Journal of Financial Economics, 82(1):227$249,2006$. 
[4] M. A. Benjamin, H. O. Hinnant, T. T. Shigeno, and D. N. Olmstead. Multi-sensor fusion, Oct. 16 2007. US Patent 7,283,904.

[5] H. Bühler. Volatility markets. consistent modeling, hedging and practical implementation. PhD Disseration, Technical University Berlin, 2006.

[6] P. Christoffersen and K. Jacobs. Which garch model for option valuation? Management science, 50(9):1204-1221, 2004.

[7] P. Christoffersen, K. Jacobs, and K. Mimouni. An empirical comparison of affine and non-affine models for equity index options. Manuscript, Springer, 2006.

[8] D. A. Coley. An introduction to genetic algorithms for scientists and engineers, volume 1655. World scientific Singapore, 1999.

[9] R. Cont and S. Ben Hamida. Recovering volatility from option prices by evolutionary optimization. 2004.

[10] B. Dumas, J. Fleming, and R. E. Whaley. Implied volatility functions: Empirical tests. The Journal of Finance, 53(6):2059-2106, 1998.

[11] B. Dupire et al. Pricing with a smile. Risk, 7(1):18-20, 1994.

[12] R. J. Elliott, L. Aggoun, and J. B. Moore. Hidden Markov models: estimation and control, volume 29. Springer Science \& Business Media, 2008.

[13] R. J. Elliott and G.-H. Lian. Pricing variance and volatility swaps in a stochastic volatility model with regime switching: discrete observations case. Quantitative Finance, 13(5):687-698, 2013.

[14] R. Gimeno and J. M. Nave. A genetic algorithm estimation of the term structure of interest rates. Computational Statistics \& Data Analysis, 53(6):2236-2250, 2009. 
[15] B. K. Grace. Black-scholes option pricing via genetic algorithms. Applied Economics Letters, 7(2):129-132, 2000.

[16] P. S. Hagan, D. Kumar, A. S. Lesniewski, and D. E. Woodward. Managing smile risk. 70+ DVDs FOR SALE \& EXCHANGE, page 249, 2002.

[17] X.-J. He and S.-P. Zhu. How should a local regime-switching model be calibrated? Submitted to Journal of Banking and Finance, 2016.

[18] X.-J. He and S.-P. Zhu. An alternative form used to calibrate the heston option pricing model. Computers \& Mathematics with Applications, 2016 (http://dx.doi.org/10.1016/j.camwa.2016.02.023).

[19] S. L. Heston. A closed-form solution for options with stochastic volatility with applications to bond and currency options. Review of financial studies, 6(2):327-343, 1993.

[20] S. Howison. Matched asymptotic expansions in financial engineering. Journal of Engineering Mathematics, 53(3-4):385-406, 2005.

[21] J. Hull and A. White. The pricing of options on assets with stochastic volatilities. The journal of finance, 42(2):281-300, 1987.

[22] S. Hwang, S. Stachell, and P. Valls Pereira. How persistent is volatility? an answer with stochastic volatility models with markov regime switching state equations. Journal of Business Finance \& Accounting, 34:1002-1024, 2007.

[23] A. Kaeck and C. Alexander. Vix dynamics with stochastic volatility of volatility. ICMA Centre, Henley Business School, University of Reading, UK, 2010.

[24] M. Kalimipalli and R. Susmel. Regime-switching stochastic volatility and short-term interest rates. Journal of Empirical Finance, 11(3):309-329, 2004. 
[25] K. G. Lim and D. Zhi. Pricing options using implied trees: Evidence from ftse-100 options. Journal of Futures Markets, 22(7):601-626, 2002.

[26] S. Mikhailov and U. Nögel. Hestons stochastic volatility model: Implementation, calibration and some extensions. John Wiley and Sons, 2004.

[27] A. Peiro. Skewness in financial returns. Journal of Banking E⿱ Finance, 23(6):847-862, 1999.

[28] L. O. Scott. Option pricing when the variance changes randomly: Theory, estimation, and an application. Journal of Financial and Quantitative analysis, 22(04):419-438, 1987.

[29] J. Shu and J. E. Zhang. Pricing s\&p 500 index options under stochastic volatility with the indirect inference method. Journal of derivatives accounting, 1(2):1-16, 2004.

[30] M. E. P. So, K. Lam, and W. K. Li. A stochastic volatility model with markov switching. Journal of Business $\&$ Economic Statistics, 16(2):244-253, 1998.

[31] E. M. Stein and J. C. Stein. Stock price distributions with stochastic volatility: an analytic approach. Review of financial Studies, 4(4):727-752, 1991.

[32] M. T. Vo. Regime-switching stochastic volatility: evidence from the crude oil market. Energy Economics, 31(5):779-788, 2009.

[33] A. E. Whalley, P. Wilmott, et al. An asymptotic analysis of an optimal hedging model for option pricing with transaction costs. Mathematical Finance, 7(3):307-324, 1997.

[34] J. B. Wiggins. Option values under stochastic volatility: Theory and empirical estimates. Journal of financial economics, 19(2):351-372, 1987.

[35] S.-P. Zhu and W.-T. Chen. A new analytical approximation for european puts with stochastic volatility. Applied Mathematics Letters, 23(6):687-692, 2010. 
[36] S.-P. Zhu and W.-T. Chen. A predictor-corrector scheme based on the adi method for pricing american puts with stochastic volatility. Computers $\mathcal{E}$ Mathematics with Applications, 62(1):1-26, 2011. 